\title{
Performance Analysis of OFDM 60GHz System and SC-FDE 60GHz System
}

\author{
Xueyan Han ${ }^{1}$, Jingjing Wang ${ }^{1, \mathrm{a}}$, Wei Shi ${ }^{1, \mathrm{~b}}$ and Xinli Dong ${ }^{2}$ \\ ${ }^{1}$ School of Information Science \& Technology, Qingdao University of Science \& Technology, Qingdao, Shandong Province, China \\ ${ }^{2}$ Qingdao Branch, China United Network Communications Corporation, China
}

\begin{abstract}
In this paper, the performance of $60 \mathrm{GHz}$ wireless communication system with $\mathrm{SC}$ and OFDM is studied, the models of OFDM $60 \mathrm{GHz}$ system and SC $60 \mathrm{GHz}$ frequency domain equalization (SC-FDE) system are established, and the bit error rate (BER) performance of OFDM $60 \mathrm{GHz}$ system and SC-FDE $60 \mathrm{GHz}$ system in $802.15 .3 \mathrm{c}$ channels is compared. The simulation results show that SC-FDE $60 \mathrm{GHz}$ system has a slight advantage over OFDM system in line-of-sight (LOS) channels, while OFDM 60GHz system has a slight advantage over SC-FDE system in non-line-of-sight (NLOS) channels. For 60GHz system, OFDM $60 \mathrm{GHz}$ system has a slight advantage over SC-FDE system in overcoming multipath fading, but the performance of both is close whether in the LOS or NLOS case.
\end{abstract}

\section{Introduction}

Currently, the high-speed interface, such as high definition multimedia interface (HDMI), Gigabit wireless local area networks(WLAN) and wireless personal area networks, have a more and more urgent requirement for Gbps-class wireless transmission technology. However, the current popular wireless high-speed communications technology, such as Ultra Broadband (UWB) wireless communication technology, generally only provides a data transfer rate of hundreds of megabits per second. $60 \mathrm{GHz}$ wireless communication technology has emerged. Because of several $\mathrm{GHz}$ wideband spectrum, up to $10 \mathrm{~W}$ transmit power, and low-cost CMOS devices[1][2][3], it is the first choice in short distance wireless communication technologies.

At present, the study of $60 \mathrm{GHz}$ wireless communication physical layer for domestic and foreign research institutions and scholars mostly focus on SCFDE and OFDM modulation. In the case of gigabit-speed transmission, the time-domain equalizer is complex. To solve this problem, [4-6] proposed SC-FDE modulation. SC-FDE can be viewed as OFDM linear precoding in special cases. The output of the receiver equalizer is inserted to compensate for linear precoding brought by the FFT matrix at the transmitter. This is the SC-FDE system. SC-FDE system has a low complexity of frequency-domain multipath channel equalizer, which is similar to OFDM. However, in the actual wireless systems, both show some different characteristics. Because of IFFT precoding at the transmitter, SC-FDE has less PAPR than OFDM. In addition, the computational complexity required for both the transmitter and receiver are different, in OFDM systems, both the transmitter and receiver should execute FFT or IFFT operations. In the SC-FDE system, the transmitter does not need to execute FFT / IFFT operations, while at the receiver, it need to perform two times of FFT / IFFT operations.

Because of OFDM system with high spectrum efficiency, which can help to achieve high-speed communications, OFDM and SC-FDE are two powerful candidate technologies that can realize gigabit transmission throughput. In 802.15.3c [7] and 802.11ad [8], both standards are chosen as the physical layer transmission modulation.802.15.3c standard divides $60 \mathrm{GHz}$ band into four channels band. The standard defines three physical layer technology, namely High Speed Interface (HSI) physical layer, Audio and Visual (AV) physical layer and SC physical layer, the first two kinds are based on OFDM modulation, SC physical layer uses SC-FDE modulation. The paper uses HSI and SC over $802.15 .3 \mathrm{c}$ standards for an example, the performance of OFDM $60 \mathrm{GHz}$ and SC-FDE system are studied and simulated.

The remaining part of the paper is organized as follows: Section 2 analyze the $60 \mathrm{GHz}$ system with OFDM modulation, and conducts Monte Carlo simulations in LOS and NLOS scenes over 802.15.3c channel model; In Section 3, the $60 \mathrm{GHz}$ system with SCFDE is analyzed, and compares bit error rate (BER) performance with $60 \mathrm{GHz}$ system with OFDM modulation; Concluding remarks are given in Section 4. 


\section{The $60 \mathrm{ghz}$ System With Ofdm Modulation}

\subsection{The $60 \mathrm{GHz}$ system model based on OFDM modulation}

The $60 \mathrm{GHz}$ system model with OFDM modulation [9] is shown in Figure 1. The specific process is as follows: after LDPC channel coding, interleaving and constellation mapping, the frequency domain signal d(n) obtain the data symbols transmitted in $\mathrm{N}$ subcarriers, denoted as $\mathrm{X}(\mathrm{k})(\mathrm{k}=0,1,2, \ldots, \mathrm{N}-1)$. After IFFT, the time domain signal $x(n), n=0,1,2 \ldots, N-1$ is obtained.

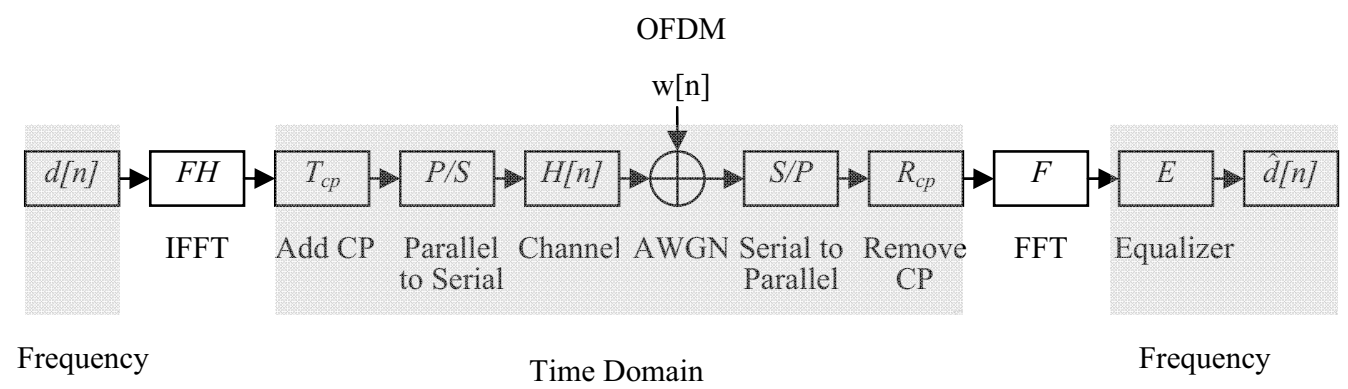

Figure 1. OFDM 60GHz system model.

$$
\begin{aligned}
& x(n)=\operatorname{IFFT}\{X(k)\} \\
& =\frac{1}{N} \sum_{k=0}^{N-1} X(k) \exp (j 2 \pi k n / N)
\end{aligned}
$$

Then the Guard Interval (GI) is added to $x(n)[10]$. GI length, denoted by TGI, is greater than the maximum delay spread of the wireless channel. The original symbol is used to cycle and expand by GI generally as the Cyclic Prefix, and by this way Inter-Carrier Interference (ICI) is suppressed, Inter-Symbol Interference (ISI) is eliminated, while the linear convolution of the channel and signal is transferred to cyclic convolution. After adding CP, the signal sequence is denoted as $x_{f}(n)$

$$
x_{f}(n)=\left\{\begin{array}{cc}
x(N+n) n= & -N_{G I},-N_{G I}+1, \mathrm{~L},-1 \\
x(n) & n=0,1, \mathrm{~L}, N-1
\end{array}\right.
$$

where NGI is the length of CP.

The signal $x_{f}(n)$ transmitted by the antenna, goes through $60 \mathrm{GHz}$ channel, then the received signal $y_{f}(n)$ is obtained. $y_{f}(n)$ is given as

$$
y_{f}(n)=x_{f}(n) * h(n)+w(n)
$$

where $h(n)$ is the sampling sequence of actual channel impulse response, $\mathrm{w}(\mathrm{n})$ is the sample sequence of additiv e white Gaussian noise with zero mean, the sampling rate is same with that of input data $\mathrm{X}(\mathrm{k})$. By removing CP fro $\mathrm{m}$ the received signal $y_{f}(n), y(n)$ is obtained,

$$
\begin{aligned}
& y(n)=y_{f}(n) \\
& n=0,1, \mathrm{~L}, N-1
\end{aligned}
$$

After FFT transform, we can obtain

$$
\begin{aligned}
& Y(k)=F F T\{y(n)\} \\
& =\sum_{n=0}^{N-1} y(n) \cdot \exp (-j 2 \pi k n / N) \\
& K=0,1 \mathrm{~L} N-1
\end{aligned}
$$

$H(k)=F F T\{h(n)\} \quad$ is the frequency domain representation of $\mathrm{h}(\mathrm{n}), \quad W(k)=F F T\{W(n)\}$ is the frequency domain representation of $\mathrm{w}(\mathrm{n})$, then we obtain

$$
\begin{aligned}
& Y(k)=X(k) H(k)+W(k) \\
& k=0,1, \mathrm{~L}, N-1
\end{aligned}
$$

After equalizing, data is through modulation constellation de-mapping, de-interleaving, channel coding translation, and finally get the decision data $\hat{d}(n)$.

\subsection{The performance simulation of OFDM $60 \mathrm{GHz}$ system}

This section chooses HSI physical layer with $802.15 .3 \mathrm{c}$ channel to study OFDM $60 \mathrm{GHz}$ system. The number of subcarriers is 512,low density parity check (LDPC) is used, BPSK, QPSK, and 16-QAM modulation modes can be chosen, 12 kinds of transmission rates of modulation and coding scheme (MCS) are provided. Table 1 lists the parameters of modulation and coding schemes.

LOS CM1.3 and NLOS CM2.3 channel are selected to simulate, and investigate bit error rate, package error rate of OFDM $60 \mathrm{GHz}$ system with different modulation and coding schemes. Each package contains $1 \mathrm{Kbit}$ information. MMSE equalization is used. 
Table 1. HSI physical layer parameters of different modulation and coding modes.

\begin{tabular}{|c|c|c|c|c|}
\hline $\begin{array}{c}\text { MCS } \\
\text { number }\end{array}$ & $\begin{array}{c}\text { Data } \\
\text { rate } \\
(\mathbf{M b} / \mathbf{s})\end{array}$ & Modulation & $\begin{array}{c}\text { Sprea } \\
\text {-ding } \\
\text { factor }\end{array}$ & $\begin{array}{c}\text { Coding } \\
\text { rate }\end{array}$ \\
\hline 1 & 1540 & QPSK & 1 & $1 / 2$ \\
\hline 2 & 2310 & QPSK & 1 & $3 / 4$ \\
\hline 3 & 2695 & QPSK & 1 & $7 / 8$ \\
\hline 4 & 3080 & $16-$ QAM & 1 & $1 / 2$ \\
\hline 5 & 4620 & $16-$ QAM & 1 & $3 / 4$ \\
\hline 6 & 5390 & 16-QAM & 1 & $7 / 8$ \\
\hline 7 & 5775 & 64-QAM & 1 & $5 / 8$ \\
\hline
\end{tabular}

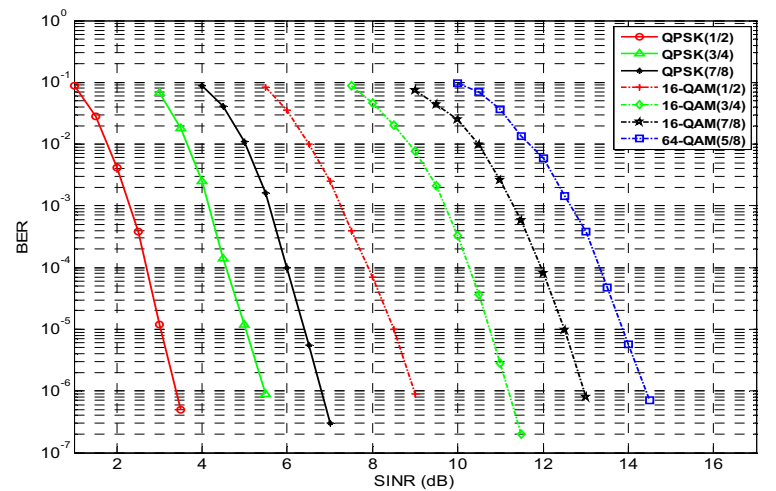

a) The BER performance simulation

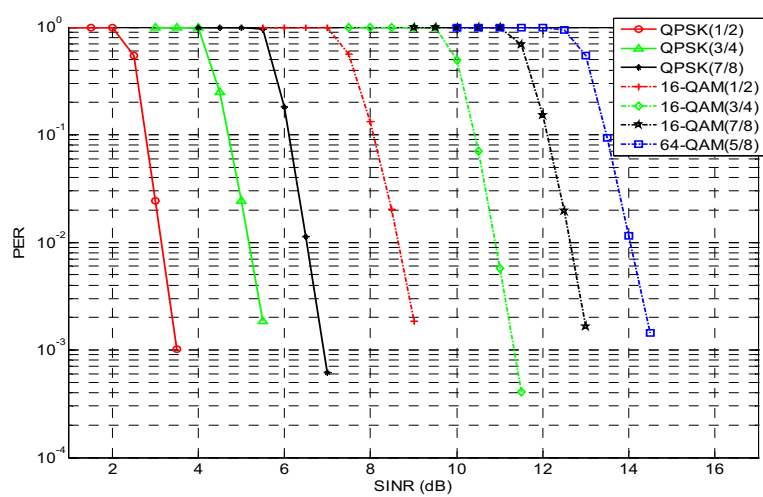

b) The PER performance simulation

Figure 2. The BER and PER performance simulation under 802.15.3c CM1.3 channel

From Figure 2 and 3, under different physical layers MCS schemes, i.e., transmission rates are $1540 \mathrm{Mb} / \mathrm{s}$, $2310 \mathrm{Mb} / \mathrm{s}, 2695 \mathrm{Mb} / \mathrm{s}, 3080 \mathrm{Mb} / \mathrm{s}, 4620 \mathrm{Mb} / \mathrm{s}, 5390 \mathrm{Mb} / \mathrm{s}$, $5775 \mathrm{Mb} / \mathrm{s}$, the SINR required for the reliable transmission with bit error rate of $10^{-6}$ under LOS CM1.3 channel of order are $3.3 \mathrm{~dB}, 5.4 \mathrm{~dB}, 6.8 \mathrm{~dB}, 9.0 \mathrm{~dB}, 11.1 \mathrm{~dB}$, $12.9 \mathrm{~dB}$ and $14.4 \mathrm{~dB}$. The SINR required for the reliable transmission with bit error rate of $10^{-6}$ under NLOS CM2.3 channel are $5.1 \mathrm{~dB}, 7.2 \mathrm{~dB}, 9.1 \mathrm{~dB}, 12.6 \mathrm{~dB}, 15.2 \mathrm{~dB}$, $17.1 \mathrm{~dB}$ and $18.7 \mathrm{~dB}$. As can be seen from the simulation, in the same modulation scheme, as the coding rate increases, the bit error rate performance will deteriorate correspondingly. In order to obtain higher transmission rates, a higher SINR requirements. In whatever rate transmission, with the same SINR, the package rate and bit error rate performance of OFDM $60 \mathrm{GHz}$ system under LOS channel has better performance than that of OFDM $60 \mathrm{GHz}$ system under NLOS channel. Comparing Figure2 (a) and Figure 3 (a), we can find that, with the increasing of coding rate and modulation order, to obtain the same error rate, the SINR difference between NLOS channel and LOS channel is growing.

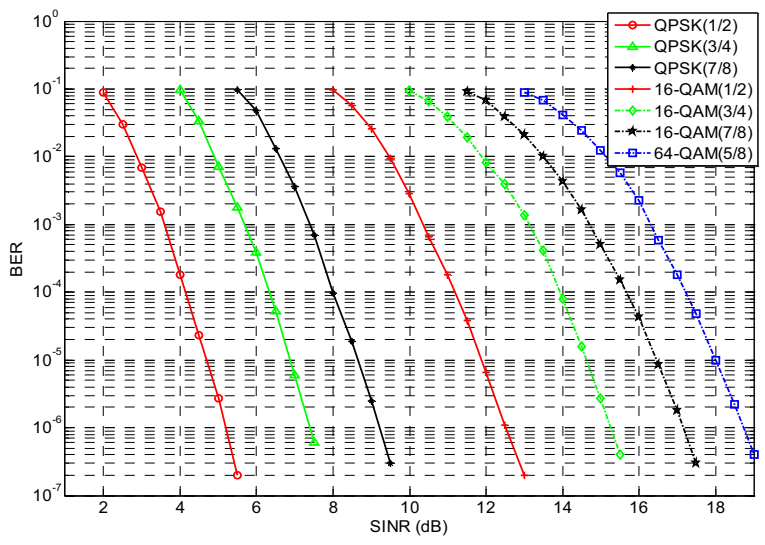

a) The BER performance simulation

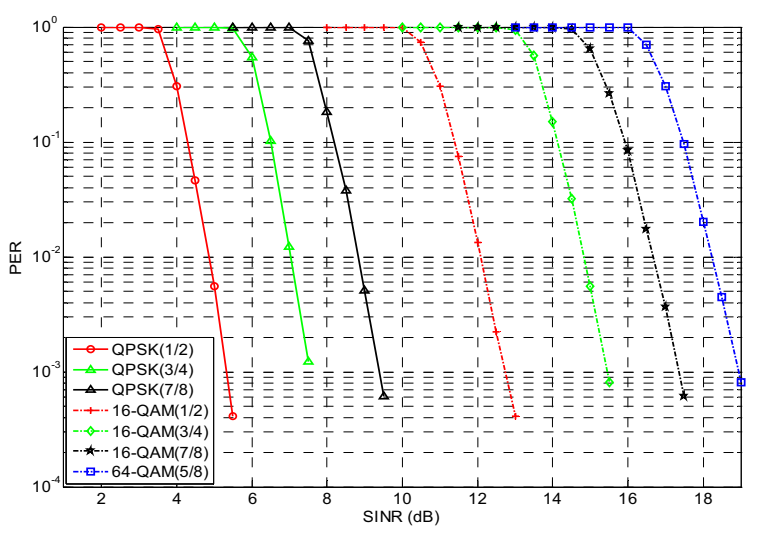

b) The PER performance simulation

Figure 3. The BER and PER performance simulation under 802.15.3c CM2.3 channel.

\section{The $60 \mathrm{ghz}$ System With Sc-Fde Modulation}

\subsection{The $60 \mathrm{GHz}$ system model based on SC-FDE modulation}

The $60 \mathrm{GHz}$ system model employing SC-FDE[11] is depicted in Figure 4 which moves the IFFT module, in transmitting end of OFDM system, to the receiving end. The same with the OFDM $60 \mathrm{GHz}$ system is that its equilibrium is also happened in frequency domain, so it is similar to the structure of the OFDM $60 \mathrm{GHz}$ system model. 


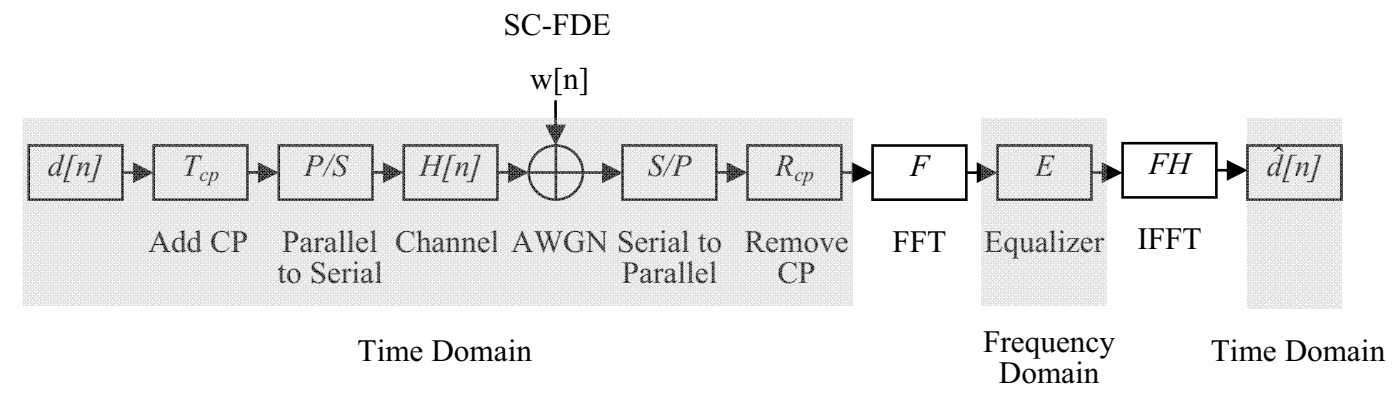

Figure 4. SC-FDE 60GHz system model.

\subsection{The performance simulation and comparison of SC-FDE $60 \mathrm{GHz}$ and OFDM system}

The study is conducted under 802.15.3c SC physical layer. When the pilot length is 64 , the data transmission rate is $1.54 \mathrm{~Gb} / \mathrm{s}-4.62 \mathrm{~Gb} / \mathrm{s}$. The sub-block length is 512 . Low Density Parity Check Code (LDPC) and Reed-Solomon code (RS) are used. BPSK, QPSK, and 16-QAM modulation modes can be chosen. The constellation mapping takes $\pi / 2$ phase shifting scheme. The modulation and coding scheme (MCS) can provide 13 kinds of transmission rate. Table 2 shows the parameters selection using the LDPC code.

Table 2. Parameter selection of modulation and coding scheme under SC physical layer.

\begin{tabular}{|c|c|c|c|c|c|}
\hline $\begin{array}{c}\text { MCS } \\
\text { Number }\end{array}$ & $\begin{array}{l}\text { Data transmission } \\
\text { rate /(Mb/s) when } \\
\text { pilot length=0 }\end{array}$ & $\begin{array}{c}\text { Data transmission } \\
\text { rate/(Mb/s) when } \\
\text { pilot length=64 }\end{array}$ & $\begin{array}{c}\text { Modulation } \\
\text { scheme }\end{array}$ & $\begin{array}{c}\text { Spreading } \\
\text { factor }\end{array}$ & Coding rate \\
\hline 7 & 1760 & 1540 & $\pi / 2$ QPSK & 1 & $\begin{array}{c}\mathrm{LDPC}(672,336) \\
\mathrm{R}=1 / 2\end{array}$ \\
\hline 8 & 2640 & 2310 & $\pi / 2 \mathrm{QPSK}$ & 1 & $\begin{array}{c}\mathrm{LDPC}(672,504) \\
\mathrm{R}=3 / 4\end{array}$ \\
\hline 9 & 3080 & 2695 & $\pi / 2$ QPSK & 1 & $\begin{array}{c}\mathrm{LDPC}(672,588) \\
\mathrm{R}=7 / 8\end{array}$ \\
\hline 13 & 5280 & 4620 & $\pi / 2-16 \mathrm{QAM}$ & 1 & $\begin{array}{c}\mathrm{LDPC}(672,504) \\
\mathrm{R}=3 / 4\end{array}$ \\
\hline
\end{tabular}

From Table 1 and Table 2, we can obtain that, when the pilot length is 64 , the data transmission rates of SC MCS7, $8 、 9 、 13$ are the same with that of HSI MCS1、2、3、5.Using the MCS in Table 1 and Table 2, under the same data transmission rates, we compare the BER performance of SC-FDE and OFDM over 802.15.3c channels.

From Figure 5(a), we can see that the performance of $\mathrm{SC}-\mathrm{FDE} 60 \mathrm{GHz}$ system is close to that of OFDM $60 \mathrm{GHz}$ system in LOS channels. With he increasing of coding rate and modulation order, the performance of SC-FDE $60 \mathrm{GHz}$ system has advantage over that of OFDM $60 \mathrm{GHz}$ system. This can be observed when SNR is large, but the advantage is small. When BER is $10^{-6}$ even with 16QAM high order modulation and high coding rate, the gap between them is less than $1 \mathrm{~dB}$. SC-FDE $60 \mathrm{GHz}$ system has a slight advantage in the high-speed transmission in LOS channels, but the performance of OFDM $60 \mathrm{GHz}$ system can be compared with that of SC-FDE system.
Figure 5(b) shows that, the performance of OFDM $60 \mathrm{GHz}$ system is better than that of SC-FDE $60 \mathrm{GHz}$ system in NLOS channels when SNR is low and QPSK (1/2LDPC) is used, and with the increasement of SNR, the performance of SC-FDE $60 \mathrm{GHz}$ system is slightly better than that of OFDM $60 \mathrm{GHz}$ system. When QPSK (3/4 LDPC) and QPSK (7/8 LDPC) are used, the performance of SC-FDE system is close to that of OFDM system. When 16QAM (3/4 LDPC) is used, the performance of OFDM system has a slight advantage over that of SC-FDE system, and the gap between them is less than $0.5 \mathrm{~dB}$ when BER is 10-6. By comparing Figure 5 , we can obtain that, SC-FDE has a slight advantage over OFDM $60 \mathrm{GHz}$ system in LOS channels, OFDM $60 \mathrm{GHz}$ system has a slight advantage over SC-FDE $60 \mathrm{GHz}$ system in NLOS channels. It shows that OFDM $60 \mathrm{GHz}$ system has a slight advantage in overcoming multipath fading, but the performance of both is close whether in the LOS or NLOS case. 


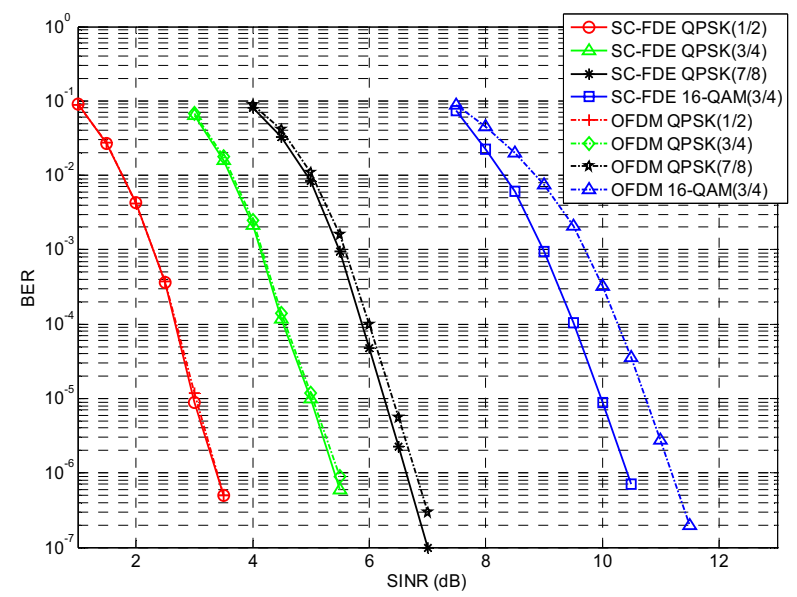

a)The BER performance simulation in CM1.3

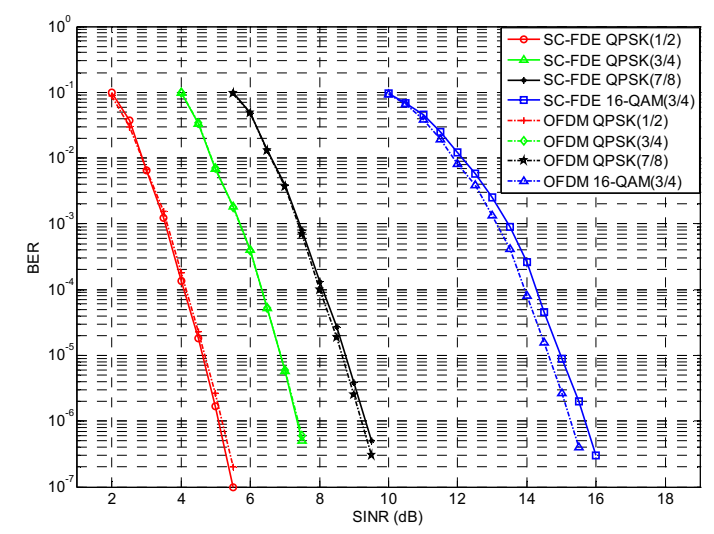

b)The BER performance simulation in CM2.3

Figure 5. The BER performance comparison of SC-FDE $60 \mathrm{GHz}$ system and OFDM $60 \mathrm{GHz}$ system under $802.15 .3 \mathrm{c}$ CM1.3 and CM2.3 channel.

\section{Conclusions}

The system models of OFDM $60 \mathrm{GHz}$ and SC-FDE $60 \mathrm{GHz}$ are established respectively, and their principle are elaborated. Physical layer communication scheme under 802.15.3c standard is analyzed, and the BER performance of OFDM $60 \mathrm{GHz}$ system and SC-FDE system in $802.15 .3 \mathrm{c}$ channels is compared and simulated respectively based on $802.15 .3 \mathrm{c}$ standard and channel model. The simulation results show that, SC-FDE has a slight advantage over OFDM in LOS channels, while OFDM has a slight advantage over SC-FDE in NLOS channels. For $60 \mathrm{GHz}$ system, OFDM has a slight advantage over SC-FDE in overcoming multipath fading, but the performance of both is close whether in the LOS or NLOS case.

\section{ACKNOWLEDGEMENTS}

The authors would like to thank the referees and editors for providing very helpful comments and suggestions.

\section{References}

1. S.K. Yong, and C.C., Chong. An overview of multigigabit wireless through millimeter wave technology: Potentials and technical challenges [J], EURASIP Journal on Wireless Communications and Networking (2007), Vol.2007, pp.1 10.

2. R.C. Daniels, and R.W. Heath, $60 \mathrm{GHz}$ wireless communications: emerging requirements and design recommendations [J], IEEE Vehicular Technology Magazine (2007), Vol.2, No.3, pp.41-50.

3. N. Guo, R.C. Qiu, S.S. Mo, and K. Takahashi. 60 $\mathrm{GHz}$ millimeter-wave radio: Principle, technology, and new results [J], EURASIP Journal on Wireless Communications and Networking (2007), Vol.2007, pp.1 8.

4. H. Sawada, Y.Shoji, C. S. Choi, K. Sato, R. Funada, H. Harada, and H. Ogawa, LOS office channel model based on TSV model. IEEE 802.15-06-0377-00- 003c, September, 2006.

5. T.Pollock, Office $60 \mathrm{GHz}$ channel measurements and model. IEEE 802.15-06-0316-00 -00-3c, July, 2006.

6. N. Moraitis, and P. Constantinou, Measurements and characterization of wideband indoor radio channel at $60 \mathrm{GHz}[\mathrm{J}]$, IEEE Transactions on Wireless Communications (2006), Vol.5, No.4, pp:880-889.

7. IEEE standards 802.15.3 $\mathrm{c}^{\mathrm{TM}}$ Part 15.3: wireless Medium Access Control (MAC) and PHYsical (PHY) layer specifications for high rate Wireless Personal Area Networks (WPANs) amendment 2: millimeterwave-based alternative physical layer extension[S]. New York, USA, IEEE Computer Society, 2009.

8. IEEE P802.11.ad/D9.0, Part 11: wireless LAN Medium Access Control (MAC) and Physical Layer (PHY) Specifications. IEEE, July, 2012.

9. A. Bourdoux, J. Nsenga, W.V.Thillo, P.Wambacq, and L.V. der Perre, Gbits/s radios @60GHz:To OFDM or not to OFDM?2008 IEEE 10th International Symposium on Spread Spectrum Techniques and Applications, 2008, pp.560-565

10. R.V. Nee, and R. Prasad, OFDM for wireless Multimedia Communications, Artech House, Boston, 2000.

11. F. Horlin, and A. Bourdoux, Digital Front-End Compensation for Emerging Wireless Systems, John Wiley\& Sons, Ltd (2007). 